Quim. Nova, Vol. 36, No. 6, 826-830, 2013

\title{
ATIVIDADES BIOLÓGICAS DOS ÓLEOS ESSENCIAIS DE Endlicheria citriodora, UMA LAURACEAE RICA EM GERANATO DE METILA
}

\author{
Klenicy K. L. Yamaguchi e Valdir F. Veiga-Junior* \\ Departamento de Química, Instituto de Ciências Exatas, Universidade Federal do Amazonas, 69077-040 Manaus - AM, Brasil \\ Tatiana do Nascimento Pedrosa, Marne Carvalho de Vasconcellos e Emerson Silva Lima \\ Faculdade de Ciências Farmacêuticas, Universidade Federal do Amazonas, 69010-300 Manaus - AM, Brasil
}

Recebido em 3/10/12; aceito em 29/1/13; publicado na web em 21/3/13

\begin{abstract}
BIOLOGICAL ACTIVITIES OF ESSENTIAL OILS OF Endlicheria citriodora, A METHYL GERANATE-RICH LAURACEAE. The essential oils of branches and leaves of Endlicheria citiodora were obtained by hydrodistillation and analysed using GC-FID, GC-MS and both NMR ${ }^{13} \mathrm{C}$ and ${ }^{1} \mathrm{H}$, resulting in the identification of methyl geranate as major constituent (93\%) in both oils. Cytotoxicity, tyrosinase-inhibition and antioxidant activities were studied and characterized. High antioxidant potential (15.52 and $13.53 \mu \mathrm{g} / \mathrm{mL}$ ), low cytotoxicity and tyrosinase inhibition (53.85\%) were observed. This is the first paper reporting the biological activities and composition of the essential oils of this species.
\end{abstract}

Keywords: linalool; tirosinase; Amazon.

\section{INTRODUÇÃO}

Lauraceae é uma família botânica conhecida por possuir espécies com interesse comercial pelos seus óleos essenciais, com aproximadamente 50 gêneros e 2500 espécies. Destas, 400 espécies distribuídas em 25 gêneros são encontradas no Brasil, possuindo grande incidência na região Amazônica. ${ }^{1-3}$

Os estudos já publicados descrevem a composição dos óleos essenciais de Lauraceae com predominância de terpenos. Este é o caso de Aniba rosaeodora, A. duckei e Cinnamomum camphora e outras espécies cujos constituintes majoritários são monoterpenos e hidrocarbonetos sesquiterpênicos, encontrados em espécies de diversos gêneros. ${ }^{4-11}$ Fenilpropanóides, como safrol, metileugenol, derivados de cinamaldeído e benzenóides ${ }^{6,12-14}$ são encontrados em espécies de Ocotea, como O. odorifera, $O$. pretiosa e $O$. sassafras.

Mesmo apresentando elevado potencial aromático em suas espécies, há muitos gêneros que não possuem descrição de seus óleos essenciais, como é o caso de Endlicheria, que tem cerca de 60 espécies distribuídas na América Tropical. ${ }^{3}$ Nos estudos envolvendo as espécies desse gênero só há relatos da composição de extratos, como o isolamento de neolignanas hexahidrobenzofuranóides (disodantina A, disodantina B, acetato de megafone, burchelina) e derivados bioativos de benzoato de benzila (2-hidróxibenzoato de benzila; 2-hidróxi-6-metóxibenzoato de benzila; 2,6-dimetóxibenzoato de benzila e 2,5-dimetóxibenzoato de benzila). ${ }^{15,16}$

O uso tradicional das plantas contra doenças de pele, especialmente para fins cosméticos, é uma prática comum na medicina popular de muitas culturas e pode fornecer pistas para a descoberta de melhores agentes despigmentantes. ${ }^{17,18}$ Formulações cosméticas produzidas a partir de ativos botânicos são utilizadas desde a antiguidade e extratos vegetais continuam desempenhando importante papel nos cosméticos contemporâneos. ${ }^{19}$ A inibição da tirosinase, enzima chave na melanogênese, é o principal mecanismo de ação de agentes despigmentantes como o ácido kójico. Assim, os pesquisadores têm se baseado nessa atividade na busca por novos agentes clareadores. ${ }^{20}$

Considerando o interesse das espécies da família Lauraceae, o presente trabalho teve como objetivo descrever a composição

*e-mail: valdirveiga@ufam.edu.br química dos óleos essenciais das folhas e dos galhos de Endlicheria citriodora e avaliar seu uso potencial em cosmético por meio da atividade antitirosinase, citotóxica, antioxidante e hemolítica desses óleos.

\section{RESULTADOS E DISCUSSÃO}

As substâncias químicas identificadas dos óleos essenciais das folhas e galhos, incluindo seus respectivos índices de retenção e porcentagens, estão sumarizadas na Tabela 1. Os óleos essenciais apresentaram um elevado rendimento: $4,29 \%$ nas folhas e $2,5 \%$ nos galhos, um alto valor comparado com os dados encontrados na literatura, em que o rendimento de 1,5\% de Aniba rosaeodora é considerado alto. ${ }^{4,8}$

Tabela 1. Composição percentual dos óleos essenciais de Endlicheria citriodora $(\%)$

\begin{tabular}{lccc}
\hline \multirow{2}{*}{ Substância } & IR & \multicolumn{2}{c}{$\begin{array}{c}\text { Concentração (\%) } \\
\text { (média } \pm \text { DP) }\end{array}$} \\
\cline { 3 - 4 } & & Folhas & Galhos \\
\hline 1. linalol & 1089 & $2,8 \pm 0,21$ & $1,2 \pm 0,05$ \\
2. geranato de metila & 1321 & $93,7 \pm 2,32$ & $95,1 \pm 1,71$ \\
3. ácido gerânico & 1391 & $1,9 \pm 0,07$ & - \\
4. $\beta$-cariofileno & 1418 & $1,5 \pm 0,02$ & - \\
5. ni & & - & $3,6 \pm 0,2$ \\
\hline Hidrocarbonetos monoterpênicos & & 2,8 & 1,2 \\
Monoterpenos oxigenados & & 95,6 & 95,1 \\
Hidrocarbonetos sesquiterpênicos & & 1,5 & - \\
Outros & & - & 3,6 \\
\hline
\end{tabular}

IR índice de retenção, ni = não identificado

Um elevado percentual da composição química dos óleos essenciais foi determinado (100\% para as folhas e $96,3 \%$ galhos), com a percentagem de monoterpenos superior a $95 \%$ em ambos os órgãos vegetais.

Em nenhum dos óleos foi detectada a presença de derivados de 
fenilpropanóides, como reportado em outros artigos para espécies do gênero Ocotea.$^{7,21,22} \mathrm{O} \beta$-cariofileno, metabólito volátil comum entre os óleos essenciais da família, foi encontrado apenas nas folhas e em pequena quantidade.

O principal constituinte foi o éster monoterpênico geranato de metila com uma percentagem elevada de $93,75 \%$ nas folhas e $95,15 \%$ nos galhos. Devido a concentração do constituinte majoritário ser alta, foi possível confirmar sua estrutura por técnicas de ressonância magnética nuclear de hidrogênio e carbono $\left(\mathrm{RMN}{ }^{1} \mathrm{He}^{13} \mathrm{C}\right)$.

$\mathrm{O}$ espectro de $\mathrm{RMN}{ }^{13} \mathrm{C}$ apresentou 11 sinais de carbonos, sendo 4 metílicos: $\delta_{\mathrm{CH}} 18,02, \delta_{\mathrm{CH}} 19,16, \delta_{\mathrm{CH}} 25,99$ e $\delta_{\mathrm{CH}} 51,11$; 2 metilênicos: $\delta_{\mathrm{CH}} 26,37$ e $\delta_{\mathrm{CH}} 41,28 ; 2$ metínicos: $\delta_{\mathrm{CH}} 115,56$ e $\delta_{\mathrm{CH}} 123,32$; e 3 sinais de carbonos não hidrogenados: $\delta_{\mathrm{CH}} 132,87$ $\delta_{\mathrm{CH}} 160,48$ e $\delta_{\mathrm{CH}} 167,63$. O espectro de DEPT $135^{\circ}$ apresentou oito sinais correspondentes aos carbonos protonados. $\mathrm{O}$ espectro de $\mathrm{RMN}{ }^{1} \mathrm{H}$ sugeriu a presença de singletos em $\delta 1,60, \delta 1,68, \delta$ 3,68 e $\delta 5,29$. Os demais sinais foram confirmados por meio do mapa de contornos HSQC onde pode-se observar as correlações entre os sinais de hidrogênio e carbono. Os valores observados nos espectros de RMN ${ }^{1} \mathrm{H}$ e ${ }^{13} \mathrm{C}$, DEPT, HSQC e a comparação com os dados espectrais relatados na literatura permitiram a confirmação da estrutura da substância majoritária. ${ }^{23}$

O geranato de metila é encontrado principalmente em frutas cítricas como o limão siciliano $(0,8 \%)$ e em espécies da família Rutaceae. Apresenta fórmula $\mathrm{C}_{11} \mathrm{H}_{18} \mathrm{O}_{2}$ com nome sistemático de 3,7-dimetil-octana-2,6-dienoato de metila ${ }^{24,25}$ e não apresenta relatos em espécies da família Lauraceae. Nos trabalhos realizados por Adams e colaboradores, ${ }^{26-28}$ o geranato de metila estava presente nos óleos essenciais em espécies de Juniperus coletados no Japão e na China e em ambos o álcool monoterpênico linalol estava presente, o que pode sugerir uma correlação entre a presença dessas substâncias. Na Figura 1 pode-se verificar a estrutura química das principais substâncias encontradas nestes óleos essenciais.

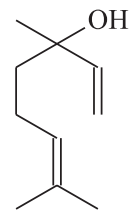

linalol

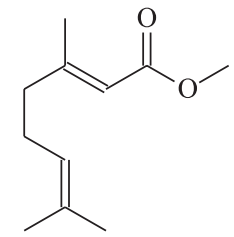

geranato de metila

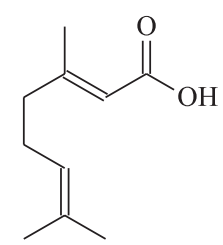

ácido gerânico

Figura 1. Constituintes principais dos óleos essenciais de Endlicheria citriodora

Os óleos essenciais são utilizados em indústrias há muitos anos devido às propriedades de suas substâncias voláteis como fonte de matérias primas. ${ }^{29,30}$ Muitas espécies pertencentes a família Lauraceae estão entre as mais utilizadas por essas indústrias, como a espécie Aniba rosaeodora que destaca-se pelo alto valor econômico. Seu óleo essencial, encontrado em grande quantidade principalmente no lenho e na casca, tem como constituinte majoritário o álcool monoterpênico linalol com rendimento de 90 a $95 \%$, utilizado como fixador de perfumes. ${ }^{4,8}$ Da mesma forma são obtidos o eugenol, de Cinnamomum zeylanicum, com rendimento de $60 \%$, e o alil benzeno safrol, extraído de Ocotea pretiosa e Sassafras albidum, com rendimentos de 80 a $85 \%$, amplamente utilizados na indústria de cosméticos. ${ }^{13,31,32} \mathrm{~A}$ principal característica do interesse nestes óleos deve-se ao fato de apresentarem um bom rendimento e um constituinte majoritário que pode ser facilmente isolado e identificado. ${ }^{33}$

O geranato de metila é um éster monoterpênico pouco conhecido. Há relatos que essa substância, aliada ao fenilacetaldeído e ao maltol, contribuem significativamente com o odor característico de batata doce, Ipomoea batatas. ${ }^{34}$ Este odor característico pôde ser verificado sensorialmente na hidrodestilação de E. citriodora.

Óleos essenciais contendo essa substância já foram relatados apresentando atividades biológicas como antimicrobiana, antifúngica e antioxidante. Entretanto, nos trabalhos reportados na literatura os rendimentos foram inferiores a $10 \% .^{24-28,35-37}$ Este, portanto, é o primeiro relato da presença dessa substância em concentração tão alta. Sua elevada taxa de biossíntese pode ser normal, uma característica da espécie, ou pode ter sido provocada por fatores bióticos ou abióticos, circadianos ou sazonais, por exemplo. Esse tipo de investigação vem sendo realizada para diversas espécies produtoras de óleos na amazônia, ${ }^{38}$ e sua influência deverá ser verificada em investigações posteriores.

Nas avaliações biológicas, o óleo essencial dos galhos apresentou capacidade antioxidante frente ao radical livre testado e inibição da enzima tirosinase. Os resultados dos testes podem ser verificados na Tabela 2.

No presente estudo, o teste de hemólise em eritrócitos de camundongos in vitro foi empregado para a avaliação da atividade hemolítica do óleo essencial obtido das folhas e galhos de E. citriodora. As concentrações estudadas variaram entre $1,22-625 \mu \mathrm{g} / \mathrm{mL}$, nas quais não foi observada hemólise, demonstrando que estes óleos possuem baixa toxicidade e sugerindo que os compostos presentes no óleo não possuem atividade hemolítica e não causam danos às membranas. A avaliação da estabilidade mecânica da membrana eritrocitária é um bom indicador de danos provocados por vários compostos como triagem de citotoxicidade. ${ }^{39} \mathrm{~A}$ cultura de células fornece uma importante ferramenta para estudo da citotoxicidade de compostos com potencial atividade terapêutica ${ }^{40}$ definindo o potencial de degeneração ou morte celular provocado pelo material constituinte nos cosméticos. Assim, resultados positivos no ensaio de citotoxicidade descaracterizam a condição de inocuidade do cosmético, sendo possível causar processos de irritabilidade nos usuários. ${ }^{41}$

A avaliação da citotoxidade dos óleos essenciais foi realizada em três linhagens celulares: NHI3T3 (fibroblastos murinos), B16F10 (melanoma murino) e, adenocacirnoma gástrico humano (ACP02) em concentração única $(50 \mu \mathrm{g} / \mathrm{mL})$ num período de $72 \mathrm{~h}$ de exposição, onde se observou baixa citotoxicidade em todas as linhagens estudadas, demonstrando que o extrato tem ação citotóxica inespecífica para células neoplásicas e normais. A tabela 2 mostra que os valores da $\mathrm{CI}_{50}$ (concentração inibitória letal de $50 \%$ das células) da doxorrubicina (controle positivo) são bastante inferiores a concentração testada do extrato. Em $50 \mu \mathrm{g} / \mathrm{mL}$ nenhuma linhagem celular chegou a $50 \%$ de citotoxicidade. A avaliação da citotoxicidade nos fornece um parâmetro da segurança na utilização desses compostos.

Tabela 2. Atividades biológicas dos óleos essenciais de Endlicheria citriodora. Os resultados estão expressos em \% de inibição. Para os testes do DPPH e tirosinase o óleo foi testado na concentração de $100 \mu \mathrm{g} / \mathrm{mL}$ e nos demais testes a $50 \mu \mathrm{g} / \mathrm{mL}$

\begin{tabular}{cccccc}
\hline & $\mathrm{DPPH} \mu \mathrm{g} / \mathrm{mL}$ & Tirosinase $\left(\mathrm{IC}_{50}\right)$ & Viab. Cel. em B16F10 (\%) & Viab. Cel. em ACP02 (\%) & Viab. Cel. em NIH3T3 (\%) \\
\hline Folhas & 15,52 & 53,85 & $72,6 \pm 3,77$ & $109,8 \pm 5,25$ & $85,3 \pm 0,46$ \\
Galhos & 13,53 & 16,92 & $75,2 \pm 9,96$ & $76,4 \pm 4,84$ & $79,8 \pm 2,58$ \\
Padrão & $11,1 \pm 0,07^{\mathrm{a}}$ & $8,9 \pm 0,11^{\mathrm{b}}$ & $0,05^{\mathrm{c}}$ & $1,57^{\mathrm{c}}$ & $0,17^{\mathrm{c}}$ \\
\hline
\end{tabular}

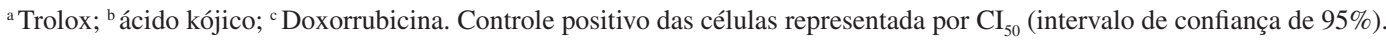


Na inibição da enzima tirosinase, o óleo essencial dos galhos apresentou uma boa inibição, no entanto, foi inferior à encontrada para o padrão, ácido kójico (Tabela 2). A inibição da tirosinase observada para os óleos presentes nas folhas $(53,8 \%)$ indica que a $\mathrm{CI}_{50}$ desse óleo essencial é pouco inferior a $100 \mu \mathrm{g} / \mathrm{mL}$, demonstrando um potencial para esta atividade. Ao compararmos nossos resultados com os relatos da literatura para outros produtos naturais, entre óleos essenciais e extratos, verificamos que as $\mathrm{CI}_{50}$ variam entre $79-1600 \mu \mathrm{g} / \mathrm{mL}$, sendo em sua maioria superiores a $100 \mu \mathrm{g} / \mathrm{mL}$, confirmando assim o potencial cosmético do óleo de E. citriodora. ${ }^{42-46}$ O uso de compostos sintéticos normalmente causa irritação na pele. Baseando-se nisso, os pesquisadores têm buscado utilizar compostos naturais no desenvolvimento de novos produtos, e várias substâncias já foram identificadas como potentes inibidores da tirosinase e da melanogênese. ${ }^{18} \mathrm{~A}$ atividade inibitória da tirosinase se dá de maneira competitiva e não-competitiva ocorrendo por diferentes mecanismos, entre os quais, podemos citar a quelação dos íons cobre da enzima ou a interferência nas etapas oxidativas do processo de formação de melanina catalisado pela tirosinase. ${ }^{20,47}$

A capacidade de sequestro do radical livre DPPH foi maior do que a encontrada em outros trabalhos com óleo essencial de espécies dessa família. ${ }^{11,48}$ Nenhum dos óleos essenciais analisados possui em sua composição compostos com reconhecida atividade antioxidante em quantidades significativas, tais como a presença de $\beta$-cariofileno associado com compostos fenólicos que têm seu potencial antioxidante aumentado devido ao efeito sinérgico, o que representa uma nova informação sobre a atividade do composto geranato de metila.

Dessa forma, a percentagem do constituinte majoritário associado ao elevado rendimento deste óleo e às atividades biológicas encontradas, propicia a utilização comercial desta espécie e a realização de novos estudos envolvendo atividades biológicas mais avançadas.

\section{PARTE EXPERIMENTAL}

\section{Material vegetal}

As folhas e galhos de Endlicheria citriodora foram coletados na Reserva Florestal Ducke, Manaus, Amazonas, em agosto de 2009. Todo o material vegetal foi obtido de um mesmo indivíduo, na fase estéril. A espécie tem exsicata depositada (INPA 191998) e foi identificada no Projeto Flora da Reserva Ducke pelos botânicos H. van der Werff e A. Vicentini.

\section{Extração dos óleos essenciais}

As amostras de folhas e galhos foram secas à sombra, reduzidas em moinho de facas e submetidas à hidrodestilação por um período de 4 h, utilizando aparelho do tipo Clevenger modificado. Os óleos essenciais extraídos foram secos com sulfato de sódio anidro, acondicionados em pequenos frascos de vidro âmbar e mantidos sob refrigeração. O cálculo do rendimento foi realizado a partir da relação da massa do óleo obtido com as massas dos materiais vegetais secos utilizados nas extrações (triplicata).

\section{Análise dos óleos essenciais}

Os óleos essenciais foram diluídos em hexano e as soluções obtidas foram submetidas à análise por cromatografia em fase gasosa com detector de ionização de chama (CG-DIC) para a análise quantitativa e determinação dos índices de retenção, e por cromatografia em fase gasosa acoplada ao espectrômetro de massas (CG-EM), para a obtenção dos espectros de massas.

\section{Análise em CG-DIC}

Os óleos foram diretamente analisados em cromatógrafo em fase gasosa modelo CG 2010 da Shimadzu ${ }^{\odot}$ com detector por ionização de chama (DIC). As análises foram realizadas com coluna CP-Sil 5 CB (100\% dimetilpolissiloxano) da Varian $^{\odot}$, com medidas de $15 \mathrm{~m}$ x $0,25 \mathrm{~mm} \times 0,25 \mu \mathrm{m}$, sendo utilizado como gás de arraste hélio (He) em fluxo de 2,0 mL/ min. A injeção em modo split 1:10 foi realizada com injetor a $250{ }^{\circ} \mathrm{C}$ e o detector de ionização de chama a $290^{\circ} \mathrm{C}$. O forno foi programado de $60^{\circ} \mathrm{C}$ a $180{ }^{\circ} \mathrm{C} \mathrm{a} 3{ }^{\circ} \mathrm{C} / \mathrm{min}$. Foram coinjetados padrões de hidrocarbonetos lineares para a determinação dos índices de retenção.

\section{Análise em CG-EM}

Após a análise por CG-DIC, os óleos foram analisados em cromatógrafo em fase gasosa modelo QP-2010 da Shimadzu ${ }^{\odot}$ com detector por espectrometria de massas (CG-EM). As análises foram realizadas com coluna VF-1MS da $\operatorname{Varian}^{\oplus}$, com medidas de $15 \mathrm{~m}$ x 0,25 mm $\mathrm{x} 0,25 \mu \mathrm{m}$. As condições da análise foram as mesmas utilizadas para CG-DIC, com temperatura do detector a $250^{\circ} \mathrm{C}$. Para a detecção foi aplicada a técnica de impacto eletrônico a $70 \mathrm{eV}$.

\section{Identificação dos constituintes dos óleos essenciais}

A determinação da composição química dos óleos essenciais foi realizada com base nos dados de tempo de retenção, obtidos por CG-DIC, e dos espectros de massas, obtidos por CG-EM. Os índices de retenção foram calculados utilizando a Equação de van der DoolKratz, relacionando os tempos de retenção das substâncias presentes nos óleos essenciais com os tempos de retenção de hidrocarbonetos lineares (série homóloga de $\mathrm{C}_{9}-\mathrm{C}_{22}$ ) que foram coinjetados com a amostra. Os índices de retenção e os espectros de massas foram comparados com dados da espectroteca Wiley 7.0 e da literatura. ${ }^{49}$

\section{Identificação do componente majoritário}

\section{Análise por RMN}

Os espectros de Ressonância Magnética Nuclear de hidrogênio $\left(\mathrm{RMN}{ }^{1} \mathrm{H}\right)$, carbono $\left(\mathrm{RMN}{ }^{13} \mathrm{C}-{ }^{1} \mathrm{H}\right.$ desacoplado) e DEPT $135^{\circ}$ foram registrados em espectrômetro da Varian modelo INOVA de 11,7 T, operando a $500 \mathrm{MHz}$ para ${ }^{1} \mathrm{H}$ e $125 \mathrm{MHz}$ para ${ }^{13} \mathrm{C}$. As amostras foram solubilizadas em $600 \mu \mathrm{L}$ de clorofórmio deuterado $\left(\mathrm{CDCl}_{3}\right)$.

\section{Ensaio do DPPH}

A determinação da atividade antioxidante foi realizada por meio da avaliação da capacidade dos antioxidantes presentes nas amostras em sequestrar o radical estável DPPH, segundo metodologia utilizada por Molyneux (2004), ${ }^{50}$ com modificações. Foram adicionados primeiramente, em cada cavidade de uma microplaca de 96 poços, $270 \mu \mathrm{L}$ de DPPH $(1 \mathrm{mg} / \mathrm{mL})$ e $30 \mu \mathrm{L}$ das soluções das amostras na concentração de $100 \mu \mathrm{g} / \mathrm{mL}$. Como controle positivo utilizou-se Trolox ${ }^{\circledR}$ e, para o controle negativo, foram utilizados $270 \mu \mathrm{L}$ de DPPH e $30 \mu \mathrm{L}$ de etanol. A placa foi incubada por $30 \mathrm{~min}$ em temperatura ambiente no escuro e realizou-se a leitura da absorbância em 517 $\mathrm{nm}$. As análises foram realizadas em triplicata e os resultados foram expressos em porcentagem de captura do radical DPPH.

\section{Teste de inibição da tirosinase}

A inibição da tirosinase foi realizada segundo método descrito por Chan et al., 2008 em triplicata. ${ }^{42}$ Preparou-se uma solução do 
óleo em DMSO (1 mg/mL). Numa microplaca de 96 poços, $20 \mu \mathrm{L}$ de cada solução dos óleos foram pipetados em triplicata e adicionados $80 \mu \mathrm{L}$ de solução de tirosinase a $0,1 \mathrm{mg} / \mathrm{mL}$. As absorbâncias 1 (A1) foram determinadas em $475 \mathrm{~nm}$. A placa foi incubada por 5 minutos a $37^{\circ} \mathrm{C}$ e em seguida adicionou-se $100 \mu \mathrm{L}$ do reagente de cor DOPA (diidroxifenilalanina) a $0,5 \mathrm{mg} / \mathrm{mL}$. A placa foi novamente incubada por 15 minutos a $37^{\circ} \mathrm{C}$ e determinou-se a absorbância final (A2) no mesmo comprimento de onda. Como controle positivo foi utilizado o acido kójico preparado da mesma forma que as amostras.

\section{Ensaio de citotoxicidade}

Para avaliação da citotoxicidade do óleo realizou-se o ensaio de Alamar Blue, metodologia descrita por Ahmed et al. (1994). ${ }^{51}$ As análises foram realizadas em triplicata utilizando as linhagens celulares NIH $3 \mathrm{~T} 3$ (fibroblastos murinos), B16F10 (Melanoma murino) e APC02 (adenocarcinoma gástrico humano). As linhagens foram cultivadas em meio DMEM (Meio Dulbecco Modificado ou Dulbecco's Modified Eagles Medium) acrescido de $10 \%$ de soro fetal bovino, $1 \%$ de penicilina e $1 \%$ de estreptomicina. Todas as células foram incubadas a $37{ }^{\circ} \mathrm{C}$ e atmosfera úmida com $5 \% \mathrm{CO}_{2}$ Uma vez tratadas com o óleo a $50 \mu \mathrm{g} / \mathrm{mL}$ e incubadas por um período de $72 \mathrm{~h}$, foram adicionados $10 \mu \mathrm{L}$ da solução de uso de Alamar Blue $0,4 \% 2$ horas antes da leitura de fluorescência (Excitação: 540 nm e Emissão: 585 nm).

\section{Avaliação do potencial hemolítico}

O teste foi realizado em placas de 96 poços usando uma suspensão a $2 \%$ de eritrócitos de camundongos em solução de $0,85 \%$ $\mathrm{NaCl}$ contendo $10 \mathrm{mM} \mathrm{CaCl}_{2}$, de acordo com método descrito por Jimenez et al. (2003), em triplicata. ${ }^{52} \mathrm{O}$ óleo foi testado em concentrações $(1,22-625 \mu \mathrm{g} / \mathrm{mL})$. Após incubação à temperatura ambiente por 30 minutos e centrifugação, o sobrenadante foi removido e a hemoglobina liberada foi mensurada por leitor de espectrofotômetro na absorbância de $540 \mathrm{~nm}$.

\section{CONCLUSÃO}

O óleo essencial de E. citriodora foi analisado pela primeira vez e, por meio de técnicas cromatográficas GC-EM, CG-DIC e RMN, identificou-se o geranato de metila como constituinte majoritário, tanto nos galhos quanto nas folhas, com teores superior a $93 \%$. O óleo essencial dos galhos apresentou atividade de inibição da enzima tirosinase e baixa capacidade hemolítica, despertando o interesse para estudos mais aprofundados destas atividades neste óleo. A família Lauraceae apresenta muitas espécies odoríferas de outros gêneros e este trabalho contribui com o conhecimento de um gênero pouco estudado cientificamente, e que se apresenta promissor devido às atividades biológicas e ao elevado rendimento do óleo essencial e de seu constituinte majoritário.

\section{AGRADECIMENTOS}

Os autores agradecem à FAPEAM, CAPES e CNPq pelo apoio financeiro.

\section{REFERÊNCIAS}

1. Cronquist, A.; An Integrated System of Classification of Flowering Plants, Columbia University Press: New York, 1981.

2. Burger, W. C.; van der Werff, H.; Fieldiana Bot. 1990, 23, 1.

3. Chanderbali, A. S. Monograph 2004, 91, 1.

4. Gottlieb, O. R.; Mors, W. B.; Bol. Inst. Quim. Agric. 1958, 53, 7.
5. Duñg, N. X.; Khiên, P. V.; Chiên, H. T.; Leclercq, P. A.; J. Essent. Oil Res. 1993, 5, 451.

6. Gottlieb, O. R.; Koketzu, M.; Magalhães, M. T.; Maia, J. G. S.; Mendes, P. H.; Rocha, A. I.; Silva, M. L.; Wilberg, V. C.; Acta Amaz. 1981, 11, 143.

7. Takaku, S.; Haber, W. A.; Setzer, W.; Biochem. Syst. Ecol. 2007, 35, 525.

8. Maia, J. G. S.; Andrade, E. H. A.; Couto, H. A. R.; Da Silva, A. C. M.; Marx, F.; Henke, C.; Quim. Nova 2007, 30, 1906.

9. Telascrea, M.; Araújo C. C. de; Cavalheiro A. J.; Marques, M. O. M.; Facanali, R.; Moraes P. L. R.; Quim. Nova 2008, 31, 503.

10. Cicció, J. F.; Chaverri, C.; Quim. Nova 2008, 31, 605.

11. Alcântara, J. M.; Yamaguchi, K. K. L.; Veiga Junior, V. F.; Lima, E. S.; Quim. Nova 2010, 33, 141.

12. Senanayake, U. M.; Lee, T. H.; Wills, R. B. H.; J. Agric. Food Chem. 1978, 26, 822

13. Rizzini, C. T.; Mors, W. B.; Botânica Econômica Brasileira, 2. ed., Âmbito Cultural: Rio de Janeiro, 1995.

14. Giang, P. M.; Koenig, W. A.; Son, P. T.; J. Nat. Med. 2006, 60, 248.

15. Ma, W. W.; Anderson, J. E.; McLaughlin, J. L.; Pharm. Biol. 1991, $29,237$.

16. Ma, W. W.; Kozlowski, J. F.; McLaughlin, J. L.; J. Nat. Prod. 1991, 54, 1153.

17. Comai, S.; Dall`Acqua, S.; Grillo, A.; Castagliuolo, I.; Gurung, K.; Innocenti, G.; Fitoterapia 2010, 81, 11.

18. Momtaz, S.; Mapunya, B. M.; Houghton, P. J.; Edgerly, C.; Hussein, A.; Naidoo, S.; Lall, N.; J. Ethnopharmacol. 2008, 119, 507.

19. Angerhofer, C. K.; Maes, D.; Giacomoni, P. U.; Skin Aging HandbookAn Integrated Approach to Biochemistry and Product Development, Willian Andrew: NewYork, 2009.

20. Chang, T. S.; Int. J. Mol. Sci. 2009, 10, 2440.

21. Ballabeni, V.; Tognolini, M.; Bertoni, S.; Bruni, R.; Guerrini, A.; Rueda, G. M.; Barocelli, E.; Pharm. Res. 2007, 55, 23.

22. Lima, L. M.; Ormelli, C. B.; Brito, F. F.; Miranda, A. L. P.; Fraga, C. A. M.; Barreiro, E. J.; Pharm. Acta Helv. 1999, 73, 281.

23. Freeman, P. K.; Siggel, L.; Chamberlain, P. H.; Clapp, G. E.; Tetrahedron 1988, 44, 5051.

24. Araújo Jr, C. P.; Dissertação de mestrado, Universidade Federal Rural de Pernambuco, Brasil, 2009.

25. Rahimi-Nasrabadi, M.; Gholivand, M. B.; Batooli, H.; J. Nanomater. Bios. 2009, 4, 819.

26. Adams, R. P.; Zhang, S.; Chu, G.; J. Essent. Oil Res. 1996, 8, 53.

27. Adams, R. P.; Thappa, R. K.; Agarwal, S. G.; Kapahi, B. K.; Srivastava, T. N.; Chaudhary, R. P.; J. Essent. Oil Res. 1998, 10, 21.

28. Adams, R. P.; Hsieh, C.; Murata, J.; Schwarzbach, A. E.; Physiol. Behav. 2011, 93, 118.

29. Marques, C. A.; Floresta e Ambiente 2001, 8,195.

30. Bizzo, H. R.; Hovell, A. M. C.; Rezende, C. M.; Quim. Nova 2009, 32, 588.

31. Takaoka, D.; Takaoka, K.; Ohshita, T.; Hiroi, M.; Phytochemistry 1976, 15,425 .

32. Lima, M. P.; Zoghbi, M. G. B.; Andrade, E. H. A.; Silva, T. M. D.; Fernandes C. S.; Acta Amaz. 2005, 35, 363.

33. Romoff, P.; Ferreira, M. J. P.; Padilla, R.; Toyama, D. O.; Fávero, O. A.; Lago, J. H. G.; Quim. Nova 2010, 33, 1119.

34. Wang, Y.; Kays, S. J.; J. Amer. Soc. Hort.Sci. 2000, 125, 638.

35. Coutinho, I. D; Cardoso, C. A. L.; Ré-Poppi, N.; Melo, A. M.; Vieira, M. C.; Honda, N. K.; Coelho, R. G.; Braz. J. Pharm. Sci. 2009, 45, 767.

36. Chu, S. S.; Liu, S. L.; Liu, Q. Z.; Liu, Z. L.; Du, S. S.; J. Med. Plant. Res. 2011, 5, 4621.

37. Gilles, M.; Zhao, J.; An, M.; Agboola, S.; Food Chem. 2010, 119, 731.

38. Barbosa, P. C. S.; Medeiros, R. S.; Sampaio, P. T. B.; Vieira, G.; Wiedemann, L. S. M.; Veiga-Junior, V. F.; J. Braz. Chem. Soc. 2012, 23, 1823.

39. Sharma, P.; Sharma J. D.; J. Ethnopharmacol. 2001, 74, 239. 
40. Vasconcellos, M. C.; Montenegro, R. C.; Militão, G. C.; Fonseca, A. M.; Pessoa, O. D.; Lemos, T. L.; Pessoa, C.; Moraes, M. O.; Costa-Lotufo, L. V.; Z. Naturf. 2005, 60, 394.

41. Chorilli, M.; Tamascia, P.; Rossim, C.; Salgado, H. R. N.; J. Basic Applied Pharm. Sci. 2009, 30, 19.

42. Chan, E. W. C.; Lim, Y. Y.; Wong, L. F.; Lianto, F. S.; Wong, S. K.; Lim, K. K.; Joe, C. E.; Lim, T. Y.; Food Chem. 2008, 109, 477.

43. Arung, E. T.; Kusuma, I. W.; Christy, E. O.; Shimizu, K.; Kondo, R.; J. Nat. Med. 2009, 63, 473 .

44. Chiari, M. E.; Joray, M. B.; Ruiz, G.; Palacios, S. M.; Carpinella, M. C.; Food Chem. 2010, 120, 10

45. Mukherjee, P. K.; Maity, N.; Nema, N. K.; Sarkar, B. K..; Phytomedicine 2011, 19, 64.

46. Wang, C. Y.; NG, C. C.; Lin, H. T.; Shyu, Y. T.; J. Biosci. Bioeng. 2011, $111,554$.
47. Heo, S. J.; Ko, S. C.; Kang, S. M.; Cha, S. H.; Lee, S. H.; Kang, D. H.; Jung, W. K.; Affan, A.; Oh, C.; Jeon, Y. J.; Food Chem. Toxicol. 2010, $48,1355$.

48. Joshi, S. C.; Mathela, C. S.; Pharmacognosy 2012, 4, 80.

49. Adams, R. P.; Identification of essential oil components by gas chromatography/ quadrupole mass spectroscopy, Allured: Card Stream I L, 2007.

50. Molyneux, P.; Songklanakarin J. Sci. Technol. 2004, 26, 211.

51. Ahmed, S. A.; Gogal, J. R. R. M.; Walsh, J. E.; J. Immunol. Methods 1994, 170, 211.

52. Jimenez, P. C.; Fortier, S. C.; Lotufo, T. M. C.; Pessoa, C.; Moraes, N. E. A.; Moraes, M. O.; Costa-Lotufo, L. V.; J. Exp. Mar. Biol. Ecol. 2003, $287,93$. 